\title{
On Combinatorial Depth Measures
}

\author{
Stephane Durocher* Robert Fraser* Alexandre Leblanc* Jason Morrison* ${ }^{*} \quad$ Matthew Skala*
}

\begin{abstract}
Given a set $P=\left\{p_{1}, \ldots, p_{n}\right\}$ of points and a point $q$ in the plane, we define a function $\psi(q)$ that provides a combinatorial characterization of the multiset of values $\left\{\left|P \cap H_{i}\right|\right\}$, where for each $i \in\{1, \ldots, n\}, H_{i}$ is the open half-plane determined by $q$ and $p_{i}$. We introduce two new natural measures of depth, perihedral depth and eutomic depth, and we show how to express these and the well-known simplicial and Tukey depths concisely in terms of $\psi(q)$. The perihedral and eutomic depths of $q$ with respect to $P$ correspond respectively to the number of subsets of $P$ whose convex hull contains $q$, and the number of combinatorially distinct bisections of $P$ determined by a line through $q$. We present algorithms to compute the depth of an arbitrary query point in $O(n \log n)$ time and medians (deepest points) with respect to these depth measures in $O\left(n^{4}\right)$ and $O\left(n^{8 / 3}\right)$ time respectively. For comparison, these results match or slightly improve on the corresponding best-known running times for simplicial depth, whose definition involves similar combinatorial complexity.
\end{abstract}

\section{Introduction}

This paper presents new work on measuring the degree to which a point $q$ is interior or central relative to a given set $P$ of $n$ points in $\mathbb{R}^{d}$, i.e., the depth of $q$ with respect to $P$. When $d=1$, natural measures for the depth of $q$ relative to $P$ include the minimum of the number of points of $P$ to the left and right of $q$, and the number of subsets of $P$ whose interval contains $q$. Both are maximized by a median of $P$. The one-dimensional median has many possible generalizations to two and higher dimensions. We focus on the case $d=2$ and examine combinatorial measures of depth, specifically those defined as functions of the number of points of $P$ contained in some half-plane through $q$. Such measures of depth include Tukey depth and simplicial depth. Our examination captures some previous definitions of depth, leads us to define two new depth measures, and provides algorithms for computing these measures of depth and the corresponding medians, defined as points of maximal depth.

In Section 2 we review results related to common notions of depth, including Tukey depth, simplicial depth,

\footnotetext{
*University of Manitoba, Winnipeg, Canada, \{durocher, fraser, mskala\}@cs. umanitoba.ca, $\{$ alex_leblanc,jason.morrison\}@umanitoba.ca
}

and half-space counts. Building on the half-space counts of Rousseeuw and Ruts [23] (see Section 2.1), we introduce the function $\psi_{j}(q): \mathbb{R}^{2} \rightarrow\{0, \ldots, n\}$ in Section 3: given any $P=\left\{p_{1}, \ldots, p_{n}\right\} \subseteq \mathbb{R}^{2}, q \in \mathbb{R}^{2}$, and $j \in\{0, \ldots, n-1\}, \psi_{j}(q)$ is the number of open halfplanes $H_{i}$ determined by $q$ and some $p_{i} \in P$ such that $\left|P \cap H_{i}\right|=j$. The $n$ values $\psi(q)=\left\langle\psi_{0}(q), \ldots, \psi_{n-1}(q)\right\rangle$ summarize pertinent combinatorial properties of the set $P$, allowing simple computation of various depth measures as functions of $\psi(q)$. Moreover, $\psi(q)$ is itself easy to calculate, and it has attractive geometric properties. Specifically, we show how to express simplicial depth and Tukey depth in terms of $\psi(q)$. We introduce two new natural measures of depth, perihedral depth in Section 4 and eutomic depth in Section 5, each defined in terms of $\psi(q)$, for which we present algorithms to compute medians in $O\left(n^{4}\right)$ and $O\left(n^{8 / 3}\right)$ time respectively, and the depth of an arbitrary query point $q$ in $O(n \log n)$ time for both. For comparison, these results match or slightly improve on the corresponding best-known running times for simplicial depth, whose definition involves similar combinatorial complexity (see Section 2.3). We show examples of these depth measures in Figure 1. The perihedral and eutomic depths of $q$ with respect to $P$ correspond respectively to the number of subsets of $P$ whose convex hull contains $q$, and the number of distinct bisections of $P$ determined by lines through $q$. These have intuitive probabilistic interpretations: after scaling by a normalizing factor (dependent on $n$, but independent of $q$ ) the perihedral depth of $q$ with respect to $P$ is equal to the probability that $q$ lies inside the convex hull of a subset of $P$ selected at random, whereas the eutomic depth of $q$ with respect to $P$ is equal to the probability that $q$ lies on a halving line of $P$. Both of these are reasonable measures of centrality, further motivating their definitions as depth measures.

\section{Related Work and Definitions}

Consider a set of $n$ points $^{1} P$ in $\mathbb{R}^{d}$ and a point $q$ in $\mathbb{R}^{d}$ in general position (specifically, no $k+1$ points of $P$ and $q$ lie on any $(k-1)$-flat for any $k \leq d)$. Let $C H(P)$ denote the convex hull of set $P$. A number of functions of the form $f(q, P):\left(\mathbb{R}^{d} \times\left(\mathbb{R}^{d}\right)^{n}\right) \rightarrow \mathbb{R}$ that define a

\footnotetext{
${ }^{1}$ Several of the depth measures described in this paper have natural generalizations to $\mathbb{R}^{d}$, in which case the definition is presented in terms of an arbitrary $d$; algorithmic results discussed apply to points in $\mathbb{R}^{2}$.
} 


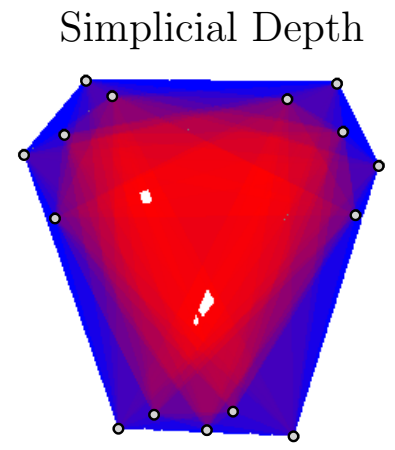

Maximum depth $=134$

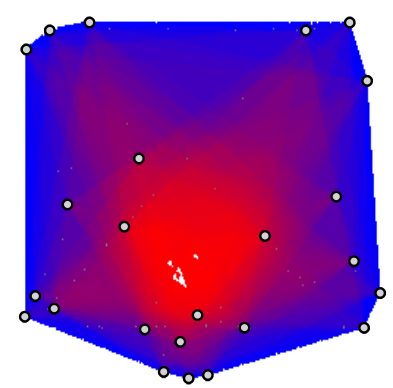

Maximum depth $=562$

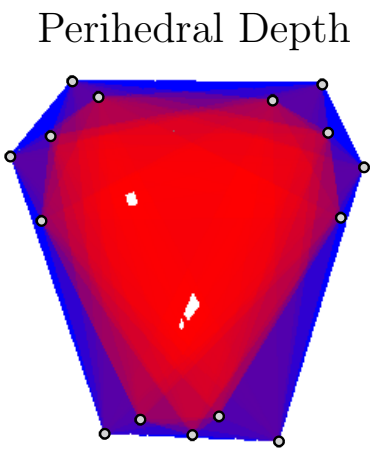

Maximum depth $=30431$

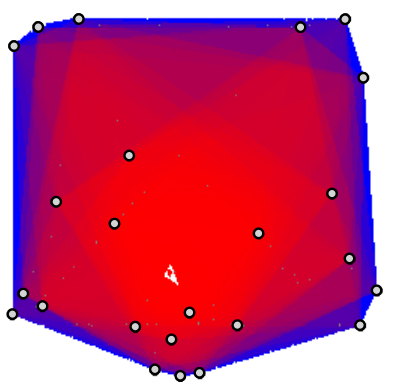

Maximum depth $=16688127$

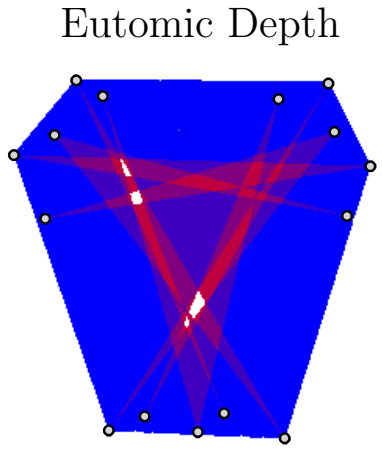

Maximum depth $=9$

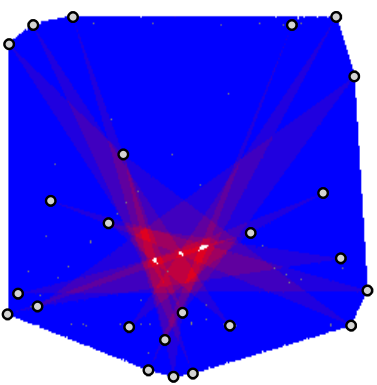

Maximum depth $=18$
Tukey Depth

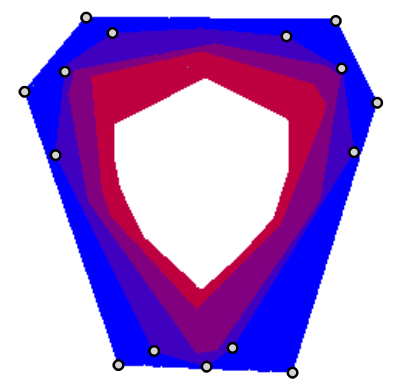

Maximum depth $=5$

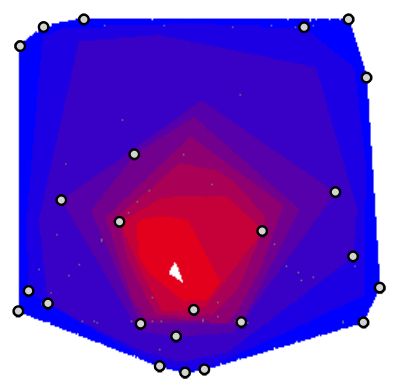

Maximum depth $=10$

Figure 1: Simplicial, perihedral, eutomic and Tukey depths are shown for two point sets. The colours progress from blue to red for increasing depth, and regions of maximum depth are shown in white. Note that in the bottom figures, the region of maximum depth is unique for each depth measure.

measure of the depth of $q$ relative to $P$ have been studied extensively. In particular, Tukey (or half-space) depth [26] and simplicial depth [17] are well understood and related to the depth measures introduced in this paper. See Sections 2.2 and 2.3. Other measures of this form are described briefly below. For additional details on depth measures, see the reviews of Aloupis [1], Small [25], and Zuo and Serfling [27].

In addition to Tukey depth and simplicial depth, several other common depth measures are primarily combinatorial. Convex hull peeling $[4,24]$ expresses the depth of $q$ as the number of times the convex hull of $P$ must be removed before $q$ appears on the hull. The ray shooting depth [21] is the minimum number of $(d-1)$-simplices on $P$ hit by any ray from $q$. For majority depth $[8,18]$, any $d$ points in $P$ determine a hyperplane and its two associated half-spaces; a half-space containing a majority of points in $P$ is called a majority side. The majority depth of $q$ is the number of majority sides of $P$ that contain $q$.

In addition to the algorithmic problem of evaluating a depth measure $f(q, P)$ for an arbitrary point $q$, the problem of identifying a point $q_{\max }$ of maximum depth with respect to a given set $P$ is typically difficult. Such a point $q_{\max }$ is a median of $P$ with respect to the given depth measure $f$, where $q_{\max }=\arg \max _{q \in \mathbb{R}^{d}} f(q, P)$.

\subsection{Half-Space Counts}

Half-space counts, denoted $h_{i}(q)$, were introduced by Rousseeuw and Ruts [23] for bivariate depth $(d=2)$, and are fundamental to previous work in computing Tukey and simplicial depths. In Section 3 we define a summary statistic of $\left\langle h_{1}(q), \ldots, h_{n}(q)\right\rangle$ which we call the $\psi$ histogram.

Consider a point $q$ whose depth is queried, and for each $i \in\{1, \ldots, n\}$, let $\alpha_{i}$ denote the angle between the vectors $p_{i}-q$ and $(1,0)$. Without loss of generality, assume the points $p_{1}, \ldots, p_{n}$ are sorted in angular order such that $0 \leq \alpha_{1}<\ldots<\alpha_{n}<2 \pi$. For each $i \in\{1, \ldots, n\}$, the half-space count $h_{i}(q)$ is the largest integer such that $\alpha_{i}<\alpha_{i+1} \leq \alpha_{i+h_{i}(q)}<\alpha_{i}+\pi$, where $\alpha_{n+j}=\alpha_{j}+2 \pi$ for all $j$. This definition of $h_{i}(q)$ is equivalent to counting the number of points of $P$ in the right open half-plane defined by the line through the points $\left(p_{i}, q\right)$ and the vector $p_{i}-q$. Given any arbitrarily ordered set $P$ of $n$ points in $\mathbb{R}^{2}$ and any $q \in \mathbb{R}^{2}$, Rousseeuw and Ruts [23] also give a method for computing $h_{i}(q)$ in $O(n)$ time for any fixed $i$, and an optimal $\Theta(n \log n)$ time algorithm for computing $\left\langle h_{1}(q), \ldots, h_{n}(q)\right\rangle$. The 
optimality of their algorithm in the real RAM model was proved by Aloupis et al. [2].

\subsection{Tukey (Half-Space) Depth}

The Tukey depth of a point $q$, denoted $T D(q, P)$, is the minimum number of points of $P$ in any closed half-space containing $q$ on its boundary. The Tukey median of $P$ is a point of maximum Tukey depth. Rousseeuw and Ruts [23] express the Tukey depth of $q \in P$ in terms of $h_{i}(q)$ as $\min _{i}\left\{\min \left(h_{i}(q), n-h_{i}(q)-1\right)\right\}$. Since their algorithm for computing all $n$ values $\left\langle h_{1}(q), \ldots, h_{n}(q)\right\rangle$ requires only $O(n \log n)$ time for any given point $q$, the computation of the Tukey depth of $q$ may be determined in the same time, which is optimal [2]. The best known algorithms for computing a Tukey median require $O\left(n \log ^{3} n\right)$ time (deterministic) [16] and $O(n \log n)$ time (randomized) [7]. Recently, Chen et al. [9] presented a randomized generalization of the algorithm of Rousseeuw and Ruts [23] for computing an approximation to the Tukey depth. Finally, Bremner et al. [5] show that the Tukey depth of a given point $q$ can be computed in $O(n+k \log k)$ time, where $k=T D(q, P)$.

\subsection{Simplicial Depth}

Every subset of $P$ of cardinality $d+1$ determines a simplex; the simplicial depth of $q$, denoted $S D(q, P)$, is the number of open simplices determined by points in $P$ that contain $q$ (some definitions use closed simplices). A point of maximum simplicial depth with respect to $P$ is a simplicial median. Aloupis et al. [2] use half-space counts to define the simplicial depth of a point in $\mathbb{R}^{2}$ :

$$
S D(q, P)=\left(\begin{array}{l}
n \\
3
\end{array}\right)-\sum_{i=1}^{n}\left(\begin{array}{c}
h_{i}(q) \\
2
\end{array}\right) .
$$

This formulation subtracts the number of simplices that do not strictly contain $q$, and again requires $O(n \log n)$ time to compute the depth of a point $q$. If a median of $P$ is not restricted to the set of input points, then there are $\Theta\left(n^{4}\right)$ combinatorially distinct regions to consider for selecting a median; the best known algorithm for computing a simplical median in this setting requires $O\left(n^{4}\right)$ time [3]. The only known lower bound related to the simplicial depth is $\Omega(n \log n)$ time to calculate it for a single point [2] (matching the upper bound). Elbassioni et al. [13] show that the simplicial depth of a given point $q$ can be computed in $O(n+k)$ time, where $k=S D(q, P)$.

\section{$3 \psi$ Histograms}

In this section we introduce a summary statistic that can be used to compute several combinatorial depth measures. Given a point $q$ and a point set $P$ in $\mathbb{R}^{2}$, we define a function $\psi(q)$ such that the Tukey depth, the simplicial depth, and the perihedral and eutomic depths of Sections 4 and 5 can all be calculated easily once $\psi(q)$ is known. Moreover, $\psi(q)$ is itself easy to calculate, and it has attractive geometric properties.

Given a set $P$ of points, a point $q$, and an integer $j, \psi_{j}(q)$ counts the number of points $p_{i}$ in $P$ for which the directed line from $q$ to $p_{i}$ has exactly $j$ points of $P$ strictly on the right side. Specifically, $\psi_{j}(q)=\sum_{i=1}^{n} I\left(h_{i}(q), j\right)$, where $I\left(h_{i}(q), j\right)$ denotes an indicator function equal to 1 when $h_{i}(q)=j$ and 0 otherwise. Thus, for any $q, \psi(q)=\left\langle\psi_{0}(q), \ldots, \psi_{n-1}(q)\right\rangle$ can be interpreted as a histogram of the $h_{i}(q)$ values. Depending on the point arrangement, $\psi_{j}(q)$ could be non-zero for any integer value of $j \in[0, n-1]$; it is zero everywhere else. Given any point $q$, it is straightforward to calculate $\psi_{0}(q), \ldots, \psi_{n-1}(q)$ by computing $h_{1}(q), \ldots, h_{n}(q)$ in $O(n \log n)$ time; a histogram could be constructed along the way or in a separate pass requiring only linear additional time, so the overall time remains $O(n \log n)$.

Since $\psi_{j}(q)$ counts the $n$ values of $h_{i}(q)$, it follows that $\sum_{j=0}^{n-1} \psi_{j}(q)=n$. Perhaps less obvious is the symmetry of $\psi(q)$, the proof of which we defer to Appendix A:

Theorem 1 If $P$ and $q$ are in general position, then $\psi_{j}(q)=\psi_{n-1-j}(q)$.

Another useful property of $\psi(q)$ is that the values $j$ for which $\psi_{j}(q)$ is non-zero are a contiguous interval of integers. The proof of this result is also deferred to Appendix A.

Theorem 2 If $P$ and $q$ are in general position, and $a \leq$ $b \leq c$ are integers such that $\psi_{a}(q)>0$ and $\psi_{c}(q)>0$, then $\psi_{b}(q)>0$.

Well-known depth measures, as well as our new ones, can be expressed concisely in terms of $\psi_{j}(q)$. In particular, the Tukey depth of a point $q$ with respect to $P$ is the least number of points in $P$ strictly on one side of a line through $q$. This value is the least element in the $h_{i}$ sequence and therefore the minimum index with a nonzero count in the histogram. This gives the following proposition.

Proposition 1 Given a set of points $P \subseteq \mathbb{R}^{2}$ and a point $q \in \mathbb{R}^{2}$, the Tukey depth of $q$ with respect to $P$ can be expressed as

$$
T D(q, P)=\min \left\{j \mid \psi_{j}(q)>0\right\} .
$$

The simplicial depth of $q$ in two dimensions with respect to $P$ is the number of triangles with vertices in $P$ that contain $q$, which can be found by subtracting the triangles that do not contain $q$ from the total of $\left(\begin{array}{l}n \\ 3\end{array}\right)$ triangles. To count exactly once each triangle that 
does not contain $q$, we count it in the unique $h_{i}$ for which one vertex is the point $p_{i}$ defining the sweep line and the other two vertices are on the right (and thus counted in $h_{i}$ ). Each $h_{i}$ corresponds to $\left(\begin{array}{c}h_{i} \\ 2\end{array}\right)$ of these triangles, giving a total of $\sum_{i=1}^{n}\left(\begin{array}{c}h_{i} \\ 2\end{array}\right)$ triangles that do not include $q$. Since $\psi_{j}(q)$ is the number of values of $i$ for which $h_{i}=j$, we can group terms in the sum and use the $\psi_{j}(q)$ values to count instead, giving the following proposition.

Proposition 2 Given a set of $n$ points $P \subseteq \mathbb{R}^{2}$ and a point $q \in \mathbb{R}^{2}$, the simplicial depth of $q$ with respect to $P$ can be expressed as

$$
S D(q, P)=\left(\begin{array}{l}
n \\
3
\end{array}\right)-\sum_{j=0}^{n-1}\left(\begin{array}{l}
j \\
2
\end{array}\right) \psi_{j}(q)
$$

In addition to Tukey depth and simplicial depth, other combinatorial depth measures might seem like natural candidates for being expressed in terms of $\psi(q)$, including majority depth [8, 18], convex hull peeling depth [4, 24], zonoid depth [20, 15], and ray shooting depth [21]. Unlike majority depth, whose value varies outside $C H(P), \psi(q)$ remains invariant for all $q$ outside $C H(P)$. Similarly, for some point $q$, the convex hull peeling depth and zonoid depth at $q$ can be altered by moving points onto the convex hull of $P$ along rays emanating from $q$ without changing $\psi(q)$. Consequently, none of majority depth, convex hull peeling depth, or zonoid depth can be expressed in terms of $\psi(q)$. Although it seems unlikely, it remains to be determined whether ray shooting depth is expressible in terms of $\psi(q)$. Depth measures whose definitions involve volumes (e.g., Oja depth [22]), distances (e.g., Mahalanobis depth [10, 27]), or curves (e.g., lens depth [19]) are not determined exclusively by the arrangement of $\left(\begin{array}{l}n \\ 2\end{array}\right)$ lines through pairs of points in $P$ and, consequently, cannot be expressed in terms of $\psi(q)$.

\section{Perihedral Depth}

Definition 1 (perihedral depth) Given a set of points $P \subseteq \mathbb{R}^{2}$ and a point $q \in \mathbb{R}^{2}$, the perihedral ("around the shape") depth of $q$, denoted $P D(q, P)$, is the number of subsets of $P$ whose convex hull contains $q$.

We assume that $P$ and $q$ are in general position. A simple brute force method for determining $P D(q, P)$ is to enumerate all subsets of $P$ and count those that contain $q$ in their convex hull. While such an algorithm would require tremendous time (there are $2^{n}$ subsets of $P$ ), it does give an idea as to the nature of the computation and the importance of using the geometric properties of $P$.
We compute $P D(q, P)$ without explicit enumeration by finding the number of subsets of $P$ whose convex hulls do not contain $q$. Consider an arbitrary subset $P^{\prime}$ of $P$. Note that $q$ is not interior to $C H\left(P^{\prime}\right)$ if and only if it is on the boundary of $C H\left(P^{\prime} \cup\{q\}\right)$. Let $Q=\left\{P^{\prime} \subseteq P \mid q\right.$ is on the boundary of $\left.C H\left(P^{\prime} \cup\{q\}\right)\right\}$. By construction, $P D(q, P)=2^{n}-|Q|$. Label the points of $P$ according to their angular ordering from the horizontal line passing through the point $q$. The empty set $\varnothing$ must be in $Q$, and any other $P^{\prime}$ uniquely determines one element of $P$ which is the counterclockwise immediate predecessor of $q$ on the boundary of $C H\left(P^{\prime} \cup\{q\}\right)$. For each $p_{i}$ let $Q_{i}=\left\{P^{\prime} \subseteq P \mid p_{i}\right.$ precedes $q$ on the boundary of $\left.C H\left(P^{\prime} \cup\{q\}\right)\right\}$. Then $Q$ is the disjoint union of $\{\varnothing\}, Q_{1}, Q_{2}, \ldots, Q_{n}$. This partition of $Q$ implies

$$
P D(q, P)=2^{n}-1-\sum_{i=1}^{n}\left|Q_{i}\right| .
$$

By construction, for $1 \leq i \leq n$, the sets in $Q_{i}$ contain only points that are to the right of the line from $p_{i}$ to $q$. However, the number of points in $P$ to the right of this line is just the half-space count $h_{i}(q)$, and so $Q_{i}$ contains exactly $2^{h_{i}(q)}$ sets, all containing $p_{i}$ but otherwise formed from those $h_{i}(q)$ other points. This gives:

Theorem 3 Given a set of $n$ points $P \subseteq \mathbb{R}^{2}$ and $a$ point $q \in \mathbb{R}^{2}$, the perihedral depth of $q$ with respect to $P$ can be expressed as

$$
\begin{aligned}
P D(q, P) & =2^{n}-1-\sum_{i=1}^{n} 2^{h_{i}(q)} \\
& =2^{n}-1-\sum_{j=0}^{n-1} \psi_{j}(q) 2^{j} .
\end{aligned}
$$

Calculating $h_{i}(q)$ for all $i$ gives $P D(q, P)$, which leads to $(3)$ in $O(n)$ additional time. As discussed in Section 3 , the complete list of values $\left\langle h_{1}(q), \ldots, h_{n}(q)\right\rangle$ can be computed in $O(n \log n)$ time and $O(n)$ space.

Consider the subsets of $P$ of cardinality $k$ for some $k \leq n$ and let $P D_{k}(q, P)$ denote the number of these subsets whose convex hull contains $q$. Observe that

$$
P D(q, P)=\sum_{k=1}^{n} P D_{k}(q, P) .
$$

Note that $P D_{3}(q, P)=S D(q, P)$, the simplicial depth of $q$. If $P D_{k}(q, P)=\left(\begin{array}{l}n \\ k\end{array}\right)$, then $q$ has Tukey depth less than $k$ because the convex hull of every subset of size $k$ contains $q$. Arguments similar to those used to derive (2) allow us to write

$$
\begin{aligned}
P D_{k}(q, P) & =\left(\begin{array}{l}
n \\
k
\end{array}\right)-\sum_{i=1}^{n}\left(\begin{array}{c}
h_{i}(q) \\
k-1
\end{array}\right) \\
& =\left(\begin{array}{l}
n \\
k
\end{array}\right)-\sum_{j=k-1}^{n-1}\left(\begin{array}{c}
j \\
k-1
\end{array}\right) \psi_{j}(q) .
\end{aligned}
$$


Computing a perihedral median, i.e., a point $\arg \max _{q \in \mathbb{R}^{2}} P D(q, P)$, is significantly more difficult than computing the depth of an arbitrary query point. The fastest known algorithm for computing a simplicial median requires $\Theta\left(n^{4}\right)$ time in the worst case [3]. Essentially a similar algorithm can also compute a perihedral median in $O\left(n^{4}\right)$ time. The $\psi$ histogram is constant within each cell in the arrangement of all the lines connecting pairs of points in $P$. When we step across an edge $l$ in the arrangement, the $h_{i}$ sequence changes by (at most) the two elements corresponding to the points that determine $l$. That constant-sized change corresponds to changing at most four elements in the $\psi$ histogram, and then in constant time we can compute the resulting change in the perihedral depth. The arrangement is of size $O\left(n^{4}\right)$ because it is determined by the pairwise intersections of $\left(\begin{array}{l}n \\ 2\end{array}\right)$ lines, and we can traverse it in $O\left(n^{4}\right)$ steps while maintaining the $h_{i}$ sequence, the $\psi$ histogram, and the perihedral depth in constant time at each step.

Theorem 4 Given a set of $n$ points $P \subseteq \mathbb{R}^{2}$ and $a$ point $q \in \mathbb{R}^{2}$, the perihedral depth of $q$ may be computed in $O(n \log n)$ time, and a perihedral median of $P$ can be found in $O\left(n^{4}\right)$ time.

If $P^{\prime}$ is a subset of $P$ chosen uniformly at random from all $2^{n}$ subsets, the probability that $q$ is interior to $\mathrm{CH}\left(P^{\prime}\right)$ is given by

$$
\begin{aligned}
& \operatorname{Pr}\left(q \text { is interior to } C H\left(P^{\prime}\right)\right) \\
= & \frac{P D(q, P)}{2^{n}} \\
= & 1-2^{-n}-\sum_{j=0}^{n-1} \psi_{j}(q) 2^{-(n-j)},
\end{aligned}
$$

providing an intuitive probabilistic interpretation of perihedral depth. This also gives a normalized formulation for perihedral depth, i.e., the depth of every point lies in $(0,1)$. An alternate interpretation of $(6)$ begins by recognizing that the probability that any point of $P$ is selected as an element of a subset $P^{\prime}$ chosen uniformly at random is $1 / 2$. If instead each point is selected independently at random with a fixed probability $\xi \in(0,1)$, the probability that $p_{i}$ is selected and that $q$ is exterior to the selected subset is $\xi(1-\xi)^{n-1-h_{i}(q)}$, since the number of points of $P$ outside the half-space determined by $q$ and $p_{i}$ is exactly $n-1-h_{i}(q)$. From this, it is easy to see that (6) generalizes to

$$
\begin{aligned}
& \operatorname{Pr}\left(q \text { is interior to } C H\left(P^{\prime}\right) ; \xi\right) \\
= & 1-(1-\xi)^{n}-\xi \sum_{j=0}^{n-1} \psi_{j}(q)(1-\xi)^{n-j-1},
\end{aligned}
$$

thus suggesting a parameterized family of normalized perihedral depths. Besides its interesting interpretation,
(7) is also central to our proof of Theorem 1 that is presented in Appendix A.

\section{Eutomic Depth}

Given a set $P$ of $n$ points in $\mathbb{R}^{2}$, a line $L$ is a halving line if it partitions $P$ into two sets whose cardinalities differ by at most one. This definition implies that for any point $q$ such that $P$ and $q$ are in general position, the line determined by $q$ and any point $p_{i} \in P$ (which we denote $L_{i}$ ) is a halving line of $P \backslash\left\{p_{i}\right\}$ if and only if $n$ is odd and $h_{r}(q)=(n-1) / 2$, or $n$ is even and $h_{i}(q) \in\{n / 2-1, n / 2\}$.

Definition 2 (eutomic depth) Given a set of $n$ points $P \subseteq \mathbb{R}^{2}$ and a point $q \in \mathbb{R}^{2}$, the eutomic ("good cutting") depth of $q$, denoted $E D(q, P)$, is the number of halving lines of $P$ among $L_{1}, \ldots, L_{n}$. That is,

$$
E D(q, P)= \begin{cases}\psi_{(n-1) / 2}(q) & \text { if } n \text { is odd } \\ \psi_{n / 2-1}(q)+\psi_{n / 2}(q) & \text { if } n \text { is even }\end{cases}
$$

Consequently, if the Tukey depth of a point $q$ is $\lfloor n / 2\rfloor$ (implying that $q$ is a Tukey median), then $q$ is also a eutomic median because the only non-zero $\psi$ values are used in computing $E D(q, P)$. Generally, the Tukey median can have Tukey depth less than $\lfloor n / 2\rfloor$ and the eutomic and Tukey medians differ.

Therefore, the eutomic depth of any point $q$ can be computed in $O(n \log n)$ time and $O(n)$ space (see Section 3 for details on computing the $\psi$ histogram). Although an analogous approach to that used for finding simplicial and perihedral medians provides a $O\left(n^{4}\right)$-time algorithm for finding a eutomic median, we describe a more efficient algorithm that runs in $O\left(n^{8 / 3}\right)$ time.

First, find the set of all near-halving lines passing through two points of $P$. Specifically, find all pairs $\left(p_{a}, p_{b}\right), a \neq b$, such that

$$
h_{a}\left(p_{b}\right) \in \begin{cases}\{(n-3) / 2,(n-1) / 2\} & \text { if } n \text { is odd, } \\ \{n / 2-2, n / 2\} & \text { if } n \text { is even. }\end{cases}
$$

These pairs can all be identified in $O\left(n^{2} \log n\right)$ time and $O\left(K_{n}\right)$ space, where $K_{n}$ is the number of such pairs, each corresponding to a near-halving line. From Dey [11] we know that $K_{n} \in O\left(n^{4 / 3}\right)$.

Consider the arrangement of lines defined by points in $P$. The $\psi$ histogram is constant throughout each cell, i.e., it can only change when crossing a line. Furthermore, since the eutomic depth of a point $q$ is defined by the central values of the $\psi$ histogram, only the lines across which the central values change are relevant. Those are exactly the near-halving lines. Where $p_{a}$ and $p_{b}$ are the points defining a near-halving line, the half-counts $h_{a}$ and $h_{b}$ both change by one between 
query points $q$ and $q^{\prime}$ on either side of the segment between $p_{a}$ and $p_{b}$ (see Theorem 1), resulting in a change of two in eutomic depth on crossing the segment (if $p_{a}$ and $p_{b}$ both move into or out of the central bin or bins) or no change (if $p_{a}$ and $p_{b}$ exchange places between the two central bins, possible only in the case where $n$ is even). Therefore the arrangement of near-halving lines partitions the plane into cells, each of which is a locus of points that have the same eutomic depth.

The arrangement of $O\left(n^{4 / 3}\right)$ near-halving lines partitions the plane into $O\left(n^{8 / 3}\right)$ cells, and can be constructed in $O\left(n^{8 / 3}\right)$ time using $O\left(n^{8 / 3}\right)$ space (e.g., [12]). To check the depth of any single point $q$ in the arrangement requires $O(n \log n)$ time and $O(n)$ space. To identify a eutomic median of $P$ it suffices to traverse the arrangement to find a cell of maximum depth. When moving from one cell to a neighbouring cell, the eutomic depth changes by either $-2,0$, or 2 , depending on the point at which the near-halving line is crossed. For each near-halving line we store the two points of $P$ that define it and the number of points of $P$ on its right side. Thus, a eutomic median can be computed in time proportional to the size of the arrangement, i.e., $O\left(n^{8 / 3}\right)$ time and $O\left(n^{8 / 3}\right)$ space.

Theorem 5 Given a set of $n$ points $P \subseteq \mathbb{R}^{2}$ and a point $q \in \mathbb{R}^{2}$, the eutomic depth of $q$ may be computed in $O(n \log n)$ time, and a eutomic median of $P$ can be found in $O\left(n^{8 / 3}\right)$ time.

\section{Discussion and Directions for Future Research}

\subsection{Properties of Combinatorial Depth Measures}

Table 1 compares properties by which depth measures are commonly evaluated. These include that a median occurs at the centre of symmetry when $P$ is centrally symmetric (P1); the depth is invariant under affine transformations (P2); the depth approaches zero as $q$ moves away from $P(\mathrm{P} 3)$; the depth is non-increasing along any ray rooted at a median (P4); the contour lines bounding adjacent regions of different depth are convex (P5); the depth of $q$ is equal under the $k$ - and $d$-dimensional definitions of the depth measure when $P$ lies in a $k$-flat of $\mathbb{R}^{d}$ for all $k<d(\mathrm{P} 6)$; and a depth measure's breakdown point, i.e., the fraction $\alpha$ of $P$ that must be displaced before the median moves away from the unperturbed points of $P(\mathrm{P} 7)$.

By definition of central symmetry about a point $q^{\prime}$, every line through $q^{\prime}$ and some point $p \in P$ is a halving line. Therefore, P1 holds for eutomic depth. P2 follows immediately from the fact that convex hulls and halving lines are invariant under affine transformations. P3 is also straightforward. As is the case for simplicial medians [6], three clusters of $n / 3$ points each demonstrate that neither P4 nor P5 hold for perihedral or eutomic depths. P6 follows from the generalizations of the definitions of perihedral and eutomic depths to higher dimensions (see below). Thus, in addition to satisfying all of the properties satisfied by simplical depth, perihedral depth also satisfies P6, while eutomic depth also satisfies both $\mathrm{P} 1$ and $\mathrm{P} 6$.

Although P1-P7 are commonly evaluated for depth measures, these provide only a limited classification of depth measures (e.g., the trivial function $f(q, P)=1$ if $q$ is in the convex hull of $P$ and 0 otherwise satisfies properties $\mathrm{P} 1-\mathrm{P} 6$ ). Furthermore, convexity and monotonicity arguably limit the ability of a depth measure to express a refined characterization of the relative position of a query point within a point set. Thus, while these properties help compare aspects of different depth measures, this classification alone is insufficient to quantify a given depth measure's ability to provide an informative high-dimensional median.

\subsection{Higher Dimensions}

The generalization of perihedral depth to $d$ dimensions follows immediately by counting the subsets of $P$ whose $d$-dimensional convex hull contains the query point $q$. Similarly, the generalization of eutomic depth to $d$ dimensions follows by counting the halving hyperplanes of $P$ through $q$.

\subsection{Query Data Structures}

Suppose the point set $P$ is known ahead of a sequence of point depth queries for perihedral or eutomic depth. Instead of computing the depth of each query point independently, requiring $O(n \log n)$ time per query, a query data structure could be constructed on $P$ to provide more efficient depth queries subsequently. The usual time-space trade-off applies, where a larger data structure permits faster query time. Thus, the respective arrangements of all lines determined by pairs of points in $P$ and all near-halving lines of $P$ can be stored using $O\left(n^{4}\right)$ and $O\left(n^{8 / 3}\right)$ space, and paired with a pointlocation query data structure (e.g., [14]) to support arbitrary depth queries in $O(\log n)$ time per query. The space requirements for these data structures are likely prohibitively large for many applications. Determining structures which use improved space (e.g., quadratic or linear in $n$ ) is an interesting direction for future research.

\subsection{Other Combinatorial Depth Measures}

As mentioned in Section 3, depth measures whose definitions involve volumes, distances, or curves cannot be expressed in terms of $\psi(q)$. Furthermore, none of majority depth, convex hull peeling depth, or zonoid depth can be expressed in terms of $\psi(q)$. It remains to be determined whether ray shooting depth (a combinatorial 


\begin{tabular}{llcccc} 
& Properties & Simplicial & Tukey & Perihedral & Eutomic \\
\hline P1 & median at centre of symmetry & unknown & $\mathcal{J}[25]$ & unknown & $\checkmark$ \\
P2 & affine invariance & $\checkmark[27]$ & $\mathcal{J}[1]$ & $\checkmark$ & $\checkmark$ \\
P3 & vanishing at $\infty$ & $\mathcal{V}[27]$ & $\mathcal{J}[1]$ & $\checkmark$ & $\checkmark$ \\
P4 & monotonicity relative to median & $\times[27]$ & $\mathcal{J}[5]$ & $\times$ & $\times$ \\
P5 & convexity of depth contours & $\times[27]$ & $\mathcal{V}[5]$ & $\times$ & $\times$ \\
P6 & consistency across dimensions & $\times$ & $\checkmark$ & $\checkmark$ & $\checkmark$ \\
P7 & breakdown point & unknown & $1 / 3^{\ddagger}[1]$ & unknown & unknown \\
\hline
\end{tabular}

Table 1: Comparing Properties of Depth Measures

$\dagger^{\dagger} \mathrm{P} 1$ is sometimes defined with respect to rotational [6] or other symmetries [27], instead of central symmetry.

$\ddagger$ The Tukey median has breakdown point $1 / 3$ in $\mathbb{R}^{2}$; the breakdown point is a function of the dimension $d$ in general.

depth measure) is expressible in terms of $\psi(q)$.

\section{References}

[1] G. Aloupis. Geometric measures of data depth. In DIMACS Series in Discrete Mathematics and Theoretical Computer Science, volume 72, pages 147$158,2006$.

[2] G. Aloupis, C. Cortes, F. Gomez, M. Soss, and G. Toussaint. Lower bounds for computing statistical depth. Comp. Stat. \& Data Analysis, 40:223$229,2002$.

[3] G. Aloupis, S. Langerman, M. Soss, and G. Toussaint. Algorithms for bivariate medians and a Fermat-Torricelli problem for lines. Comp. Geom.: Theory \& App., 26(1):69-79, 2003.

[4] V. Barnett. The ordering of multivariate data. J. Royal Stat. Soc. Ser. A (Gen.), pages 318-355, 1976.

[5] D. Bremner, D. Chen, J. Iacono, S. Langerman, and P. Morin. Output-sensitive algorithms for Tukey depth and related problems. Stat. 8 Comp., 18(3):259-266, 2008.

[6] M. A. Burr, E. Rafalin, and D. L. Souvaine. Simplicial depth: An improved definition, analysis, and efficiency for the finite sample case. In Proc. $C C C G$, pages 136-139, 2004.

[7] T. M. Chan. An optimal randomized algorithm for maximum Tukey depth. In Proc. ACM-SIAM SODA, pages 430-436, 2004.

[8] D. Chen and P. Morin. Approximating majority depth. Comp. Geom.: Theory $\&$ App., 46(9):10591064, 2013.

[9] D. Chen, P. Morin, and U. Wagner. Absolute approximation of Tukey depth: Theory and experiments. Comp. Geom.: Theory \& App., 46(5):566$573,2013$.
[10] R. De Maesschalck, D. Jouan-Rimbaud, and D. L. Massart. The Mahalanobis distance. Chemometrics $\mathscr{E}$ Intel. Lab. Sys., 50(1):1-18, 2000.

[11] T. K. Dey. Improved bounds for planar $k$-sets and related problems. Disc. \& Comp. Geom., 19(3):373-382, 1998.

[12] H. Edelsbrunner and L. J. Guibas. Topologically sweeping an arrangement. J. Comp. Es Sys. Sci., 38(1):165-194, 1989.

[13] K. M. Elbassioni, A. Elmasry, and K. Makino. Finding simplices containing the origin in two and three dimensions. Int. J. Comp. Geom. \& Appl., 21(5):495-506, 2011.

[14] D. G. Kirkpatrick. Optimal search in planar subdivisions. SIAM J. Comp., 12(1):28-35, 1983.

[15] G. Koshevoy and K. Mosler. Zonoid trimming for multivariate distributions. Annals Stat., pages 1998-2017, 1997.

[16] S. Langerman and W. Steiger. Optimization in arrangements. In Proc. STACS, volume 2607 of LNCS, pages 50-61. Springer, 2003.

[17] R. Liu. On a notion of data depth based upon random simplices. Annals Stat., 18:405-414, 1990.

[18] R. Y. Liu and K. Singh. A quality index based on data depth and multivariate rank tests. J. ASA, 88(421):252-260, 1993.

[19] Z. Liu and R. Modarres. Lens data depth and median. J. Nonparam. Stat., 23(4):1063-1074, 2011.

[20] K. Mosler and R. Hobert. Data analysis and classification with the zonoid depth. DIMACS Series Disc. Math. \&5 Theor. Comp. Sci., 72:49, 2006.

[21] N. H. Mustafa, S. Ray, and M. Shabbir. Rayshooting depth: Computing statistical data depth of point sets in the plane. In Proc. ESA, pages 506-517. Springer, 2011. 
[22] H. Oja. Descriptive statistics for multivariate distributions. Stat. \&3 Prob. Let., 1:327-332, 1983.

[23] P. J. Rousseeuw and I. Ruts. Bivariate location depth. J. Royal Stat. Soc. Ser. C (App. Stat.), 45(4):516-526, 1996.

[24] M. I. Shamos. Geometry and statistics: Problems at the interface. Technical report, DTIC Document, 1976.

[25] C. G. Small. A survey of multidimensional medians. Int. Stat. Review, 58(3):263-277, 1990.

[26] J. Tukey. Mathematics and the picturing of data. In Proc. Int. Cong. Math., pages 523-531, 1975.

[27] Y. Zuo and R. Serfling. General notions of statistical depth function. Annals Stat., 28(2):461-482, 2000 .

\section{A Appendix: Proofs for Properties of $\psi(q)$}

In this Appendix we present proofs of both theorems presented in Section 3.

Proof. [of Theorem 1] Consider the point $q$ for which the histogram $\psi(q)=\left\langle\psi_{0}(q), \ldots, \psi_{n-1}(q)\right\rangle$ is to be constructed. Recall that $\psi(q)$ is the histogram of the halfspace counts $h_{i}(q)$, where each $h_{i}(q)$ is the number of points of $P$ in the right open half-plane determined by the line through the points $p_{i}$ and $q$, and the vector $p_{i}-q$.

Now, let $g_{i}(q)$ denote the number of points of $P$ in the left open half-space defined by the line through the points $p_{i}$ and $q$, and the vector $p_{i}-q$. By construction, $g_{i}(q)=n-h_{i}(q)-1$. We denote the histogram of the left half-space counts as $\Psi(q)=\left\langle\Psi_{0}(q), \ldots, \Psi_{n-1}(q)\right\rangle$, whose elements satisfy $\Psi_{j}(q)=\psi_{n-1-j}(q)$ for $j=$ $0,1, \ldots, n-1$. We next prove that $\Psi_{j}(q)=\psi_{j}(q)$, implying the stated symmetry of the $\psi$ histogram.

To see this, consider the probability that the query point $q$ is interior to $C H\left(P^{\prime}\right)$ when the subset $P^{\prime}$ is constructed by selecting each point of $P$ with probability $\xi \in(0,1)$ independently of all others. Working from the (right) half-space counts $h_{i}(q)$, this probability was derived in (7) in terms of the $\psi$ histogram. Working from the left half-space counts, the symmetry of the problem implies that

$$
\begin{aligned}
& \operatorname{Pr}\left(q \text { is interior to } C H\left(P^{\prime}\right) ; \xi\right) \\
= & 1-(1-\xi)^{n}-\xi \sum_{j=0}^{n-1} \Psi_{j}(q)(1-\xi)^{n-j-1},
\end{aligned}
$$

is also valid. These two equations together imply, after letting $t=1-\xi$, that

$$
\sum_{j=0}^{n-1}\left[\Psi_{j}(q)-\psi_{j}(q)\right] t^{n-j-1}=0,
$$

for all $t \in(0,1)$. The left hand side of this equality is a polynomial in $t$ that is uniformly equal to zero on the unit interval, implying that its coefficients all have to be zero, in turn implying that $\Psi_{j}(q)=\psi_{j}(q)$ for $j=$ $0,1, \ldots, n-1$. (This follows, for instance, by considering all derivatives of the polynomial.)

Proof. [of Theorem 2] Sweeping a ray from $q$ counterclockwise through a circle, pausing when it reaches each point in $P$ to record the number of elements of $P$ strictly to the right of the line containing the ray, gives a cyclic sequence of $n$ integers which are the half-space counts $h_{i}$. Let $h(\theta)$ be the number of points in $P$ strictly to the right of the line containing the ray as a function of the ray's angle. This function increases by one every time the ray encounters a point, and decreases by one every time another ray from $q$ in the opposite direction (the other half of the line) encounters a point. General position implies that no two of these events coincide. In the complete sweep, the line encounters each point once on each side, so that $h(\theta)$ returns to its starting value. Only the values immediately after each increase are recorded in the sequence $h_{i}$.

If $a, b$, and $c$ are not all distinct then the theorem is trivially true, so the interesting case is when $a<b<c$. Having $\psi_{a}(q)$ and $\psi_{c}(q)$ both non-zero means that the number of points to the right of the ray must have assumed both the values $a$ and $c$ during the sweep. Then because $h(\theta)$ can only increase or decrease by one at a time, it must have also assumed all integer values between $a$ and $c$, notably including $b$. Moreover, if we choose the starting point for the cyclic sweep such that $h(\theta)=a$ is true before $h(\theta)=b$, then there must be a first time it assumes the value $b$ after $a$. If we consider the first time it reaches $b$ after reaching $a$, and the last time it reaches $a$ before that, then between those points $h(\theta)$ must be strictly between $a$ and $b$. The move immediately prior to $h(\theta)=b$ must be an increase. Therefore on that occasion $b$ will be included in the $h_{i}$ sequence and counted in the histogram, and so $\psi_{b}(q)>0$. 